\title{
Infertility etiologies are genetically and clinically linked with other diseases in single meta-diseases
}

\author{
Juan J Tarín ${ }^{1 *}$, Miguel A García-Pérez ${ }^{2,3}$, Toshio Hamatani $^{4}$ and Antonio Cano ${ }^{5,6}$
}

\begin{abstract}
The present review aims to ascertain whether different infertility etiologies share particular genes and/or molecular pathways with other pathologies and are associated with distinct and particular risks of later-life morbidity and mortality. In order to reach this aim, we use two different sources of information: (1) a public web server named DiseaseConnect (http://disease-connect.org) focused on the analysis of common genes and molecular mechanisms shared by diseases by integrating comprehensive omics and literature data; and (2) a literature search directed to find clinical comorbid relationships of infertility etiologies with only those diseases appearing after infertility is manifested. This literature search is performed because DiseaseConnect web server does not discriminate between pathologies emerging before, concomitantly or after infertility is manifested. Data show that different infertility etiologies not only share particular genes and/or molecular pathways with other pathologies but they have distinct clinical relationships with other diseases appearing after infertility is manifested. In particular, (1) testicular and high-grade prostate cancer in male infertility; (2) non-fatal stroke and endometrial cancer, and likely non-fatal coronary heart disease and ovarian cancer in polycystic ovary syndrome; (3) osteoporosis, psychosexual dysfunction, mood disorders and dementia in premature ovarian failure; (4) breast and ovarian cancer in carriers of BRCA1/2 mutations in diminished ovarian reserve; (5) clear cell and endometrioid histologic subtypes of invasive ovarian cancer, and likely low-grade serous invasive ovarian cancer, melanoma and non-Hodgkin lymphoma in endometriosis; and (6) endometrial and ovarian cancer in idiopathic infertility. The present data endorse the principle that the occurrence of a disease (in our case infertility) is non-random in the population and suggest that different infertility etiologies are genetically and clinically linked with other diseases in single meta-diseases. This finding opens new insights for clinicians and reproductive biologists to treat infertility problems using a phenomic approach instead of considering infertility as an isolated and exclusive disease of the reproductive system/hypothalamic-pituitary-gonadal axis. In agreement with a previous validation analysis of the utility of DiseaseConnect web server, the present study does not show a univocal correspondence between common gene expression and clinical comorbid relationship. Further work is needed to untangle the potential genetic, epigenetic and phenotypic relationships that may be present among different infertility etiologies, morbid conditions and physical/cognitive traits.
\end{abstract}

Keywords: Cancer, Disease, Gene expression, Molecular pathways, Phenomics

\section{Background}

Infertility [Unified Medical Language System (UMLS) identification (ID): C0021359] is the inability to conceive for at least one year after trying and having unprotected sex [1]. It is estimated that about $15 \%$ of couples are infertile [2]. Male and female infertility account for about a third of infertility cases, respectively, whereas the other

\footnotetext{
*Correspondence: tarinjj@uv.es

${ }^{1}$ Department of Functional Biology and Physical Anthropology, Faculty of Biological Sciences, University of Valencia, Burjassot, Valencia 46100, Spain Full list of author information is available at the end of the article
}

third of cases is because of either problems in both partners or unknown reasons [1].

Between $15-30 \%$ of infertile men and $\approx 10 \%$ of infertile women display genetic abnormalities, including chromosome aberrations, single- or multiple-gene mutations, and polymorphisms (for review, see [3]). Infertile men and women may also display mitochondrial and epigenetic disturbances, although the incidence of these defects has not been yet established (for review, see [3]). In addition to these genetic/epigenetic backgrounds, many infertile subjects are exposed to environmental toxicants/endocrine 
disruptors and/or display immunological and/or endogenous hormonal disturbances that not only may jeopardize reproductive health but also later-life morbidity and mortality.

Recently, a public web server (http://disease-connect.org) named DiseaseConnect has been created $[4,5]$. This server is focused on the analysis of common genes and molecular mechanisms shared by diseases by integrating comprehensive omics and literature data. This is a novel approach that aims to search common pathogenic mechanisms that link diseases together in a single meta-disease instead of the traditional approach of splitting the phenome (i.e., the set of phenotypes attributable to sequence variation in the human genome and specific environmental influences) into discrete entities called diseases [6].

Noteworthy, the utility of DiseaseConnect server for biologists to study mechanism-based disease connectivity was validated by Liu et al. [4]. In particular, these authors showed that diseases with shared molecular mechanisms are "likely" to be linked with clinical comorbidity data and to have the same drug treatment. However, as this validation was based on probabilistic grounds, a univocal correspondence between common gene expression and clinical comorbid relationship cannot be expected. In other words, common gene expression associations reported by DiseaseConnect web server [5] do not necessarily imply the presence of clinical comorbid relationships. In addition, clinical comorbid relationships may appear or not simultaneously in life. For instance, diseases typically characteristic of older post-reproductive adults such as senile dementia and Alzheimer's disease manifest much later in life than infertility. In contrast, diabetes mellitus or urinary tract infection may manifest concomitantly or even before infertility is evidenced. Thus, it is not possible to discriminate from DiseaseConnect clinical comorbid relationships whether infertility is linked with other diseases because it shares genes and molecular pathways with those diseases or because it results from the morbid effects of other preexisting pathological conditions. Moreover, infertility is a heterogeneous condition with multiple genetic, epigenetic, mitochondrial, immunological, hormonal and environmental etiologies [3]. Therefore, it is not possible to infer that all the infertility etiologies share the same abnormalities in gene expression and/or infertility-linked pathologies. Instead, we may expect that each particular cause of infertility has its own genetic and/or comorbid relationships with other diseases.

The present review aims to ascertain whether different infertility etiologies share particular genes and/or molecular pathways with other pathologies and are associated with distinct and particular risks of later-life morbidity and mortality. In order to reach this aim, we use two different sources of information: (1) DiseaseConnect web server for the analysis of common genes and molecular mechanisms shared by diseases; and (2) a literature search focused on clinical comorbid relationships of infertility etiologies with only those diseases appearing after infertility is manifested. We perform this literature search because DiseaseConnect web server [5] does not discriminate between pathologies emerging before, concomitantly or after infertility is manifested.

\section{Methods}

As mentioned above, this study used data on common genes and molecular mechanisms shared by infertility etiologies and other diseases from DiseaseConnect web server [5]. In addition, we performed a literature search aimed to find clinical comorbid relationships between infertility etiologies and other diseases appearing later in life, based on publications up to December 2014 identified by PubMed database using the following key words: polycystic ovary syndrome (PCOS), premature ovarian failure (POF), diminished ovarian reserve (DOR), endometriosis, fertility drugs, idiopathic/unexplained/unknown infertility, cancer, cardiovascular disease, morbidity, mortality, semen quality, oligozoospermia, teratozoospermia, asthenozoospermia and normozoospermia. We also carried out a manual search to explore the references cited in the primary articles.

The PubMed and manual literature search showed that the majority of studies did not determine whether cancer risk is associated with infertility itself or with the exposure of infertile women to fertility drugs. Notwithstanding, recent non-systematic reviews [7], systematic reviews $[8,9]$, and systematic reviews and meta-analyses [10-12] suggest that the use of fertility drugs does not increase the risk of breast, ovarian, endometrial and cervical cancer. In contrast, some studies report increased risks of borderline (low malignant potential) ovarian tumors in women treated with fertility drugs for in-vitro fertilization (IVF) (for systematic reviews, see [8,9]). These findings, however, are likely a consequence of surveillance bias and younger age of subfertile women (for systematic review, see [8]). Thus, the association between causes of infertility and breast-gynecological cancers reported in the present review can be attributed to infertility etiology in itself, not to the use of fertility drugs.

Hypergeometric test was applied by DiseaseConnect [5] to assess the significance of the Genome-Wide Association (GWAS), Online Mendelian Inheritance in Man (OMIM) and differentially expressed genes (DEG) genes shared by specific infertility etiologies and particular diseases. Hypergeometric test calculates the statistical significance of having drawn $\mathrm{k}$ successes (out of $\mathrm{n}$ total draws, without replacement, wherein each draw is either a success or a failure) from a finite population of size $\mathrm{N}$ containing exactly $\mathrm{K}$ successes. Tables 1, 2, 3 and 4 show the hypergeometric Pvalues that quantify the strength of the disease-disease connection in the number of shared genes. 
Table 1 Relation of male infertility (UMLS ID: C0021364) with other diseases that significantly share GWAS/OMIM/ DEG genes [5]

\begin{tabular}{ll}
\hline Diseases & P value $^{\mathbf{a}}$ \\
\hline Infertility & $<0.0001$ \\
Mutation abnormality & 0.0004 \\
Malignant neoplasm of urinary organ, unspecified & 0.0010 \\
Bladder neoplasm & 0.0010 \\
Urologic neoplasms & 0.0010 \\
Carcinoma of bladder & 0.0010 \\
Neoplasm of unspecified nature of bladder & 0.0010 \\
Urinary bladder diseases & 0.0011 \\
B cell lymphomas & 0.0029 \\
Liposarcoma, myxoid & 0.0036 \\
Liposarcoma & 0.0037 \\
Neoplasms, fibrous tissue & 0.0042 \\
Juvenile dermatomyositis & 0.0044 \\
Amyopathic dermatomyositis & 0.0044 \\
Fibrosarcoma & 0.0044 \\
Neoplasms, connective tissue & 0.0050 \\
Liposarcoma, dedifferentiated & 0.0058 \\
Frontotemporal lobar degeneration & 0.0074 \\
Lymphoma, non Hodgkin & 0.0077 \\
TDP 43 proteinopathies & 0.0098 \\
Myositis & 0.0099 \\
\hline A hypergeometic test was appled by DisaseConnet & \\
\hline
\end{tabular}

${ }^{\mathrm{a} A}$ hypergeometric test was applied by DiseaseConnect [5] to assess the significance of the GWAS/OMIM/DEG genes shared by male infertility and a particular disease.

\section{Genetic and clinical associations of male infertility with other diseases}

Male infertility (UMLS ID: C0021364) is a term used if a man has not been able to get a woman pregnant after at least one year of trying [1]. In the context of assisted reproductive technology (ART), the Centers for Disease Control and Prevention [13] define male factor as any cause of male infertility due to low sperm count or problems with sperm function that makes it difficult for a sperm to fertilize an oocyte under normal conditions.

Male infertility (UMLS ID: C0021364) shares genes with other pathologies (Table 1). To date, a total of 168 GWAS/OMIM/DEG genes have been found to be significantly associated with male infertility [5]. These genes are involved in ribosome, pancreatic cancer and proteasome pathways [Kyoto Encyclopedia of Genes and Genomes (KEGG) ID: hsa03010, hsa05212 and hsa03050, respectively]. These pathways play an important role in metabolism, genetic information processing (DNA replication, repair and transcription, RNA degradation and translation, and protein folding, sorting and degradation), environmental information processing, cellular processes and
Table 2 Relation of PCOS (UMLS ID: C0032460) with other diseases that significantly share GWAS/OMIM/DEG genes [5]

\begin{tabular}{ll}
\hline Diseases & P value $^{\mathbf{a}}$ \\
\hline Ovarian cysts & $<0.0001$ \\
Gonadal dysgenesis & 0.0002 \\
Leydig cell hypoplasia, type II & 0.0015 \\
Donohue syndrome & 0.0015 \\
Sulfocysteinuria & 0.0015 \\
Primary hypogonadism & 0.0015 \\
Diabetes mellitus, insulin resistant, & 0.0015 \\
with acanthosis nigricans & \\
Sulfite oxidase deficiency & 0.0015 \\
Ovarian hyperstimulation syndrome & 0.0015 \\
Rabson Mendenhall syndrome & 0.0015 \\
Eunuchism & 0.0030 \\
Pseudohermaphroditism & 0.0030 \\
ACTH deficiency, isolated & 0.0030 \\
Isolated lutropin deficiency (disorder) & 0.0030 \\
Lassa fever & 0.0031 \\
Arenaviridae infections & 0.0031 \\
Hemorrhagic fevers, viral & 0.0032 \\
Precocious puberty & 0.0046 \\
Liposarcoma, dedifferentiated & 0.0057 \\
Gonadal dysgenesis, 46,XX & 0.0061 \\
46, XX disorders of sex development & 0.0061 \\
Synovial sarcoma & 0.0067 \\
Multiple system congenital anomalies not elsewhere & 0.0076 \\
Medicine Clinical Terms (SNOMED-CT) & \\
\hline A hyperture of & \\
\hline
\end{tabular}

${ }^{\mathrm{a} A}$ hypergeometric test was applied by DiseaseConnect [5] to assess the significance of the GWAS/OMIM/DEG genes shared by PCOS and a particular disease.

human diseases such as cancer, drug resistance, substance dependence, and endocrine, metabolic, cardiovascular, immune, infectious (bacterial, viral and parasitic) and degenerative diseases [14]. Not surprisingly, male infertility is associated with increased risk of testicular (for review, see [15] and for systematic review, see [16]) and high-grade prostate (for review, see [17]) cancer. In addition, men undergoing an infertility evaluation with two or more semen abnormalities (including semen volume, concentration and motility) have higher risks of death than men with normal semen parameters after controlling for baseline comorbidity [18]. The risk adjustment for baseline comorbidity performed by Eisenberg et al. [18] deserves special mention. Actually, the presence of medical disorders such as obesity (and underweight), diabetes mellitus, metabolic syndrome, hypertension and bacterial/viral infections not only may impair semen quality/production [19] (for review, see [20] and for systematic reviews and 
Table 3 Relation of POF (UMLS ID: C0085215) with other diseases that significantly share GWAS/OMIM/DEG genes [5]

\begin{tabular}{lc}
\hline Diseases & P value $^{\mathbf{a}}$ \\
\hline Hypergonadotropic ovarian failure, X linked & $<0.0001$ \\
Premature menopause & $<0.0001$ \\
Fragile X tremor/ataxia syndrome & $<0.0001$ \\
POF7 (disorder) & $<0.0001$ \\
Spermatogenic failure 8 & $<0.0001$ \\
Hypoaldosteronism & $<0.0001$ \\
Sex chromosome disorders & $<0.0001$ \\
Adrenal cortical hypofunction & $<0.0001$ \\
Fragile X syndrome & $<0.0001$ \\
Adrenal gland hypofunction & 0.0001 \\
Adrenal gland diseases & 0.0005 \\
POF5 & 0.0008 \\
POF6 & 0.0008 \\
POF2b & 0.0008 \\
Sex chromosome aberrations & 0.0008 \\
Ptosis & 0.0016 \\
Uterine prolapse without mention of vaginal wall prolapse & 0.0016 \\
Eyelid diseases & 0.0024 \\
Blepharophimosis & 0.0040 \\
Blepharoptosis & 0.0071 \\
\hline
\end{tabular}

${ }^{\mathrm{a}} \mathrm{A}$ hypergeometric test was applied by DiseaseConnect [5] to assess the significance of the GWAS/OMIM/DEG genes shared by POF and a particular disease.
Table 4 Relation of endometriosis (UMLS ID: C0014175) with other diseases that significantly share GWAS/OMIM/ DEG genes [5]

\begin{tabular}{lc}
\hline Diseases & P value $^{\mathbf{a}}$ \\
\hline Female genital diseases & $<0.0001$ \\
Uterine diseases & $<0.0001$ \\
Myocardial infarction & $<0.0001$ \\
Coronary artery disease & $<0.0001$ \\
Sleep disorders, intrinsic & $<0.0001$ \\
Parasomnia & $<0.0001$ \\
Myocardial ischemia & $<0.0001$ \\
Dementia due to specified medical condition & $<0.0001$ \\
Restless legs syndrome & 0.0002 \\
Diabetes mellitus, non-insulin dependent & 0.0005 \\
Sex reversal, female, with dysgenesis of kidneys, & 0.0016 \\
adrenals, and lungs & \\
$46, X X$ testicular disorders of sex development & 0.0016 \\
Disorder of pancreatic internal secretion & 0.0017 \\
Hyperandrogenism & 0.0032 \\
HIV infections & 0.0044 \\
Carotid atherosclerosis & 0.0063 \\
Pancreatic intraductal papillary mucinous adenoma & 0.0074 \\
\hline Aortic eneurysm, abdominal & 0.0095
\end{tabular}

${ }^{\mathrm{a} A}$ hypergeometric test was applied by DiseaseConnect [5] to assess the significance of the GWAS/OMIM/DEG genes shared by endometriosis and a particular disease. meta-analyses, see $[21,22]$ ) but also may increase morbidity and/or mortality in subsequent years. Furthermore, deficiencies in certain nutrients such as vitamins $\mathrm{C}$ and $\mathrm{E}$, folate, selenium and zinc, and lifestyle habits including substance abuse (e.g., alcohol, cocaine and cannabis) and smoking may reduce sperm quality (for review, see [20] and for systematic review and meta-analysis, see [23]) and life expectancy (for reviews, see [24-27]). Notwithstanding, recent studies have found no significant effect of smoking and alcohol consumption on motile sperm concentration [28] and sperm morphology [29]. In addition, the use of cannabis seems to affect only sperm morphology [29].

\section{Genetic and clinical associations of female infertility etiologies with other diseases}

Female infertility (UMLS ID: C0021361) is a term used when a woman has not been able to get pregnant after at least one year of trying [1]. In the context of ART, female infertility is split into several etiologies including tubal factor, ovulatory dysfunction, diminished ovarian reserve, endometriosis and uterine factor [13]. Likewise, DiseaseConnect web server [5] segregates female infertility into several etiologies including PCOS, POF and endometriosis (see below). In contrast, literature provides studies that analyze the association of "overall" female infertility with later-life morbidity and mortality without taking into account infertility etiologies. The majority of these studies report increased risks of ovarian cancer in infertile women, especially when they remain nulliparous (for reviews, see $[9,30])$. Moreover, childless women that eventually have a child after $\geq 5$ years of self-reported infertility have higher risks of cardiovascular events (hospitalization or death caused by coronary heart disease, stroke or heart failure) during the subsequent 11.9 years after giving birth than women reporting no history of infertility that conceive within 12 months of trying [31].

In addition, mortality risk for a woman whereas it is pregnant or within 42 days of termination of pregnancy is higher in IVF pregnancies than in the general population $[32,33]$. This is most likely to be associated with the advanced reproductive age and higher incidence of multiple pregnancy and Cesarean section exhibited by IVF women [33]. Moreover, IVF women display a higher prevalence of hypertension and likely stroke during the following 8.6 years after delivery compared with primiparous control mothers from the general population [34]. However, other authors $[32,33]$ have reported that all-cause mortality risks of treated and non-treated IVF women are significantly 
lower than those of the general female population of the same age. Similarly, women who give birth after fertility therapy have fewer cardiovascular events, all-cause mortality, subsequent depression, alcoholism, and self-harm than control women from the general population who deliver without receiving fertility therapy [35]. These findings may be explained by the healthier condition/behavior and/or higher socio-economic status of infertile women compared with their general population counterparts $[30,35,36]$.

\section{PCOS}

PCOS (UMLS ID: C0032460) is a complex and heterogeneous disease influenced by many environmental and genetic factors (for review, see [37]). It accounts for $90-95 \%$ of infertile women suffering from ovulatory dysfunction attending infertility clinics in U.S. (for review, see [38]). According to the Rotterdam criteria [39], PCOS is diagnosed if a woman meets any two of the following three criteria: (1) oligo- and/or anovulation; (2) clinical and/or biochemical signs of hyperandrogenism; and (3) polycystic ovaries on ultrasounds and exclusion of other known disorders of hyperandrogenemia (congenital adrenal hyperplasias, androgen-secreting tumors and Cushing's syndrome).

DiseaseConnect web server [5] reports no molecular pathways associated with this condition. However, 52 protein complexes are significantly linked with the 23 GWAS/ OMIM/DEG genes related with PCOS [5]. Twenty four of these 52 complexes are members of the ubiquitin E3 ligase family. These complexes are involved in protein deubiquitination and ubiquitination, proteasomal ubiquitindependent protein catabolism, protein amino acid autophosphorylation, dephosphorylation and phosphorylation, response to DNA damage stimulus, mitotic cell cycle and cell aging [40]. In addition, PCOS shares genes with other pathologies including gonadal dysgenesis, precocious puberty and type 2 diabetes mellitus (Table 2).

Epidemiological studies show that PCOS is associated with increased risks of insulin resistance, impaired glucose tolerance, gestational diabetes, type 2 diabetes mellitus and metabolic syndrome (cited by [41]). Consequently, PCOS is linked with higher risk of non-fatal stroke and possibly non-fatal coronary heart disease (for systematic review and meta-analysis, see [42]).

As genetic factors play an important role in the etiology of PCOS with an estimated heritability of $65 \%$ [43], not surprisingly several studies $[44,45]$ report that mothers, fathers and brothers of women with PCOS are more likely to have cardiovascular disease in later adult life. These studies, however, did not find a significant relationship between PCOS women and a family history of diabetes. On the contrary, Louwers et al. [46] evidenced that mothers (but not fathers) above age 60 years of patients with PCOS display increased all-cause mortality, most likely as result of type 2 diabetes mellitus.
It appears that the prolonged exposure to high levels of estrogen associated with progesterone deficiency/resistance in PCOS women [47] may induce hyperplasia and uncontrolled differentiation of the endometrium. Accordingly, the probability of random mutation and DNA replication errors leading to development of cancer may be increased. In fact, PCOS is associated with higher risk of endometrial and likely ovarian cancer although not breast cancer (for review, see [48], for systematic review, see [9] and for systematic reviews and meta-analyses, see $[49,50])$. Moreover, the insulin resistance and hyperinsulinemia exhibited by PCOS women may lead to excessive secretion of androgens, luteinizing hormone (LH) and insulin-like growth factor I (IGF-I) which are additional risk factors for endometrial and ovarian cancer (for reviews, see [51,52]). Noteworthy, among the various PCOS phenotypes, those displaying hyperandrogenism have the highest metabolic morbidity and prevalence of cardiovascular risk factors including obesity/ overweight, hypertension, insulin resistance, dyslipidemia and metabolic syndrome [41,53] (for review, see [54]).

\section{POF}

POF (UMLS ID: C0085215) is a condition characterized by sustained (4 months or more) amenorrhea, elevated early-follicular levels of follicle stimulating hormone (FSH) and low estrogen levels in women less than 40 years of age (for review, see [55]). In contrast to natural menopause, women diagnosed with POF may undergo unpredictable ovarian function leading to intermittent and unpredictable menses in $50 \%$ of cases, and conceive and deliver a child in $\approx 5$ to $10 \%$ of cases. For this reason, other authors redefine this condition as 4 months or more of disordered menses (amenorrhea, oligomenorrhea, polymenorrhea, or metrorrhagia), and use the term primary ovarian insufficiency (POI) instead of POF (for review, see [56]).

POF is a multifactorial condition including multiple causes such as idiopathic, X-chromosome alterations, autosomal genetic disorders, autoimmune, infections and iatrogenic following treatment with immunosuppressant drugs, surgery for gynecological disorders or chemotherapy/pelvic irradiation for malignancy (for reviews, see $[55,57,58])$. The incidence of POF is estimated to be $1 \%$ in women under the age of $40,0.1 \%$ under 30 and $0.01 \%$ under 20. Nevertheless, the prevalence of POF is continuously increasing due to the higher survival following malignant disease (for review, see [57]).

Like in PCOS, no molecular pathways associated with POF have been reported so far by DiseaseConnect web server [5]. Only the protein complex, leukemia inhibitory factor (LIF) receptor (LIFR)-LIF-glycoprotein 130 (gp130), which is involved in interleukin receptor signaling pathway and differentiation of the nervous system 
[40], is significantly linked with the 12 GWAS/OMIM/ DEG genes of POF reported by DiseaseConnect [5]. Moreover, POF shares genes with other pathologies such as X-linked hypergonadotropic ovarian failure, adrenal gland diseases and uterine prolapse (Table 3).

Epidemiological studies show that POF women have increased risk of osteoporosis, psychosexual dysfunction, mood disorders and dementia as well as raised risk for all-cause mortality including cancer, cardiovascular disease and other causes (e.g., neurological, respiratory, renal, liver disease, diabetes mellitus, etc.). On the contrary, it is associated with lower risk of breast and endometrial cancer due to the reduction in levels of endogenous estrogens $([59,60]$ and references quoted therein; for reviews, see $[57,61])$.

It is thought that some changes in the cardiac and vascular structure and physiology that take place in menopause are due to the reduced levels of estrogen and relatively increased serum androgens associated with this condition (for review, see [62]). The early and prolonged loss of ovarian $17 \beta$-estradiol $\left(\mathrm{E}_{2}\right)$ in POF women may lead to a doubled lifetime risk for dementia and a five-fold increase risk of mortality from neurological disorders (for review, see [63]). In addition, low $E_{2}$ levels are involved in the typical loss of bone calcium and osteoporosis, although the concurrent increase in circulating FSH (i.e., the increased ratio of FSH to $\mathrm{E}_{2}$ ) is likely the main responsible for bone resorption (for review, see [64]). Likewise, an increase in the ratio between mitogenic [gonadotropinreleasing hormone (GnRH) and gonadotropins] and differentiative (sex steroids) hormones may lead to aberrant cell proliferation and cancer (for review, see [64]).

\section{DOR}

DOR is another cause of infertility that shares many features and etiologies with POF/POI. It is defined as a decreased number and/or quality of oocytes (for review, see [65]), or when the ability of the ovary to produce oocytes is reduced because of congenital, medical or surgical causes, or advanced age [13]. In women less than 35 years of age, DOR is the cause of infertility that contributes more than others to the relatively low success percentages observed in ART cycles, especially in fresh autologous cycles. In particular, the 2012 national clinic summary report by the Society for Assisted Reproductive Technology (SART) [66] shows that the live-birth percentage per fresh autologous cycle in DOR women is only $30.3 \%(542 / 1789)$ versus $36.6 \%(1135 / 3102)$ in multiple female factors, $37.2 \%(929 / 2498)$ in other factors, $38.3 \%(988 / 6580)$ in female and male factors, $40.2 \%$ $(1116 / 2776)$ in tubal factor, $41.2 \%(184 / 447)$ in uterine factor, $41.8 \%$ (813/1946) in endometriosis, $42.1 \%$ (1796/ $4265)$ in ovulatory dysfunction, $42.4 \%(2358 / 5562)$ in unknown factor, and 44.6\% (4325/9697) in male factor.
DOR has multiple causes including genetic, environmental, autoimmune, idiopathic, advanced age and iatrogenic (ovarian surgery, chemotherapy and radiation) (for reviews, see $[55,65,67])$. DOR women present regular menses and alterations of ovarian reserve tests, such as increased early follicular FSH levels, lower anti-Müllerian hormone serum concentrations, decreased antral follicular count, high dehydroepiandrosterone (DHEA)-sulfate (DHEAS) levels and low testosterone concentrations due to DHEA conversion insufficiency, especially in women over 40 years of age [68] (for review, see [55]). The prevalence of DOR in women attending infertility centers in U.S. has been steadily increasing from 10\% in 2003 to $17 \%$ in 2012 [66] (note that these percentages include women suffering only from DOR. Figures may be higher if women with multiple infertility factors were taken into account).

DiseaseConnect web server [5] does not provide any information about this particular condition. However, epidemiological studies indicate that DOR women $\leq$ 35 years of age exhibit higher levels of several cardiovascular disease risk markers including insulin resistance, C-reactive protein, triglyceride and high-density lipoprotein compared with women with normal ovarian reserve [69]. A link between DOR and breast and ovarian cancer has been established in carriers of BRCA1/2 mutations [these genes are members of the ataxia-telangiectasia mutated (ATM)-mediated DNA double-strand break repair family of genes that act via a homologous recombination mechanism]. In fact, women carrying mutations in $B R C A 1$ exhibit lower ovarian reserve evaluated on the basis of lower serum concentration of anti-Müllerian hormone levels than women with no BRCA1/2 mutations or carrying $B R C A 2$ mutations irrespectively of the presence [70] or the absence [71] of a personal history of breast or ovarian cancer. Moreover, BRCA1-deficient women have higher risk of breast and ovarian cancer ([72,73] and references quoted therein), low response to exogenous ovarian stimulation [74] and may experience earlier natural menopause [75-77]. Notwithstanding, a recent study has reported that $B R C A 1$ mutation carriers do not display a higher risk of earlier natural menopause when compared with their non-carrier relatives [78].

According to Oktay et al. [79], the function of DNA repair genes such as BRCA1 declines with age. This decrease causes accumulation of lethal DNA double-strand breaks in primordial follicles which may activate apoptotic cell death mechanisms in oocytes/follicles to prevent propagation of resultant severe mutations. Such an age-associated decline in DNA-repair function is exacerbated in BRCA1-mutant carriers. For this reason, these women display a lower ovarian reserve compared with women without BRCA1/2 mutations [70]. Of note, the effects on ovarian reserve and risk of breast and ovarian cancer in BRCA2-mutant carriers is less pronounced 
and/or delayed compared with BRCA1-mutant carriers because of a much later decrease in $B R C A$ function. Nonetheless, women with a BRCA2 mutation, not only have increased lifetime risks of developing breast and ovarian cancer like BRCA1-mutant carriers, they also exhibit a higher incidence of pancreatic cancer compared with the general population ([73] and references quoted therein).

\section{Endometriosis}

Endometriosis (UMLS ID: C0014175) is an estrogendependent, inflammatory, chronic disorder characterized by the presence of endometrial-like glands and stroma outside the uterine cavity and musculature, mainly located on the pelvic peritoneum, ovaries and the retrocervical septum (also incorrectly named rectovaginal septum [80]). Nevertheless, it can be found less commonly outside the pelvis at most sites of the body including the lungs, skin, lymph nodes, breast and brain (for reviews, see $[81,82])$. Women with endometriosis may be asymptomatic or, depending on the site of the lesions, may suffer from dysmenorrhea, pelvic pain (particularly during menstruation), dyspareunia, and/or pain associated with urination or bowel movements (for review, see [83]). Endometriosis is uncommon before menarche and usually regresses after menopause (for review, see [84]). The prevalence of endometriosis is 25$30 \%$ among infertile women (for review, see [84]) and up to $90 \%$ among reproductive women with chronic pelvic pain (for review, [85]). The etiology of endometriosis is still unknown but it is thought it is influenced by genetic, epigenetic, hormonal, environmental and immunological factors (for reviews, see [81,83,86-88]).

To date, a total of 24 GWAS/OMIM/DEG genes have been reported by DiseaseConnect web server [5] to be significantly associated with this condition. These genes are involved in vitamin $B_{6}$ metabolism pathway (KEGG pathway ID: hsa00750), which plays an important role in metabolism, genetic/environmental information processing, cellular processes and human diseases [7]. Endometriosis shares genes with other pathologies including myocardial infarction, coronary artery disease, sleep disorders, restless legs syndrome and type 2 diabetes mellitus (Table 4).

Endometriosis does not induce a catabolic state, metabolic disturbances or death like a malignancy, but it shares common characteristic of ovarian cancer including invasion, unrestrained growth, angiogenesis, decreased number of cells undergoing apoptosis, and genetic, epigenetic and biochemical alterations (for reviews, see $[82,83,85,89,90]$ ). Although the direct progression of endometriosis lesions to ovarian neoplasms has been only rarely demonstrated (for review, see [90]), literature shows a significant association of endometriosis with clear cell and endometrioid histologic subtypes of invasive ovarian cancers, and likely low-grade serous invasive ovarian cancer, melanoma and non-Hodgkin lymphoma (for reviews, see [81,82,85,91-93]).

\section{Unexplained infertility}

Unexplained (also named unknown or idiopathic) cause of infertility (UMLS ID: C0404585) is a diagnostic category used when no cause of infertility is found in either the woman or the man [13]. Despite many researchers and institutions have exerted substantial effort and invested considerable financial resources to improve infertility diagnosis, the incidence of unexplained infertility in couples attending infertility centers in U.S. has been kept more or less constant since 2003 (11-13\%) [66].

Like in DOR, DiseaseConnect web server [5] does not include the term unexplained, unknown or idiopathic infertility in its repertoire and, therefore, it does not provide any information on this condition. Nonetheless, epidemiological studies show that, in addition to endometrial cancer [94], this infertility condition is linked to ovarian cancer (for review, see [91]). In particular, serous borderline, serous invasive, endometrioid and clear cell histological subtypes of ovarian cancer [95]. It has been hypothesized that these women may display abnormal regulation of inflammatory responses by prostaglandins [95] or suffer from preexisting cancer [94] or an underlying pathology associated with both reduced fertility and increased cancer risk [96].

\section{Concluding remarks}

This review has found evidence that different infertility etiologies not only share particular genes and/or molecular pathways with other pathologies but they have distinct clinical relationships with other diseases appearing later in life. The present data endorse the principle that the occurrence of a disease (in our case infertility) is non-random in the population [6] and suggest that different infertility etiologies are genetically and clinically linked with other diseases in single meta-diseases. This finding opens new insights for clinicians and reproductive biologists to treat infertility problems using a phenomic approach instead of considering infertility as an isolated and exclusive disease of the reproductive system/hypothalamic-pituitary-gonadal axis.

We should emphasize that we have used two different sources of information to find this evidence: (1) DiseaseConnect web server [5] to search for common genes and molecular mechanisms shared by infertility etiologies and other diseases; and (2) a literature search focused on clinical comorbid relationships of infertility etiologies with only those diseases appearing after infertility is manifested. Of note, the present study does not show a univocal correspondence between common gene expression 
and clinical comorbid relationship. That is to say, not all the common gene expression associations between infertility etiologies and other diseases reported by DiseaseConnect web server [5] are translated into a clinical comorbid relationship based on the literature search we have performed. Likewise, Liu et al. [4] found that diseases with shared molecular mechanisms are likely (but not univocally) linked with clinical comorbidity data. In particular, they performed an elegant validation analysis plotting the fraction (y axis) of those mechanism-based disease-disease connections that overlap with disease comorbidity connections of the same disease pairs against various statistical significance (P-value) thresholds ( $\mathrm{x}$ axis) used to select significant disease-disease connections. The P-value of the connection between two diseases was assessed by a hypergeometric test on shared genes derived from several data sources [GWAS, OMIM, DEG, Gene Reference Into Function (GeneRIF) and disease-gene relationships from literature corpus mining (GeneWays)]. This analysis showed an overall trend for two diseases with stronger mechanism-based connections to be more likely to have a significant clinical comorbidity relationship.

It is important to bear in mind that DiseaseConnect web server [5] is not a static and complete source of information. According to Liu et al. [4], it will be continuously updated because of the rapid accumulation of disease-related omics and text literature data. Thus, the gene-expression data we have extracted from DiseaseConnect web server [5] should not be considered definitive and conclusive.

\section{Future prospects}

The novel approach linking diseases together in a single meta-disease may embrace other non-morbid phenotypic traits. In order for these traits to be included in a given infertility meta-disease, they should share genes with the particular infertility etiology that nucleates that meta-disease and/or at least one disease connected (sharing genes) with that infertility etiology.

Of note, non-morbid phenotypic traits resulting from secondary disturbances induced by a particular infertility condition and/or its associated pathologies cannot be included in this phenomic approach. For instance, PCOS shares genes with "diabetes mellitus, insulin resistant, with acanthosis nigricans" (UMLS ID: C0342278) (Table 2), a condition characterized by insulin resistance and hyperinsulinemia [97]. These metabolic/endocrine disorders may induce PCOS women to manifest cutaneous disarrangements including acanthosis nigricans (sign of insulin resistance) and hirsutism, acne and hair loss (signs of hyperinsulinemia-induced hyperandrogenism) [note that insulin stimulates the ovarian theca cells to secrete androgens and also inhibits hepatic production of sex hormonebinding globulin (SHBG), by this means elevating free testosterone levels (for review, see [52]).
Phenomics is a field of research that is still in its infancy and no clear genetic associations between non-morbid phenotypic traits and infertility etiologies have been yet established. Notwithstanding, several studies have reported significant associations between reproductive and physical/cognitive traits suggesting the presence of phenomic connections between traits. In particular, (1) endometriosis has been related with severe teenage acne [98], leanness, lower body mass index, high sensitivity to sun exposure and pigmentary traits such as natural red hair color, light eyes, nevi and freckles [99-101] (for review, see [102]). (2) Reproductive success [number of living children and (number of living children + (number of living grandchildren)/2)] has been positively correlated with high voice pitch and handgrip strength in indigenous Ovahimba (the last semi-nomadic people of Namibia) women [103]. (3) Sperm concentration, count and motility have been positively correlated with general intelligence (estimated by a $g$ factor extracted from 5 well-validated cognitive tests evaluating broad spatial, quantitative and verbal abilities) [104]. And (4) semen quality has been positively correlated with facial attractiveness of men aged 18 to 36 years when facial attractiveness is evaluated by both men and women aged 18 to 25 years in the context of a potential long-term partner [105] (not in the context of a short-term sexual partner evaluated by women [106]).

Further work is needed to untangle the potential genetic, epigenetic and phenotypic relationships that may be present among different infertility etiologies, morbid conditions and physical/cognitive traits.

\section{Abbreviations}

ART: Assisted reproductive technology; ATM: Ataxia-telangiectasia mutated; DEG: Differentially expressed genes; DHEA: Dehydroepiandrosterone; DHEAS: Dehydroepiandrosterone-sulfate; DOR: Diminished ovarian reserve; $\mathrm{E}_{2}$ : 17ß-estradiol; FSH: Follicle stimulating hormone; GeneRIF: Gene Reference Into Function; GnRH: Gonadotropin-releasing hormone; GeneWays: Disease-gene relationships from literature corpus mining; GWAS: Genome-Wide Association; ID: Identification; IGF-I: Insulin-like growth factor I; IVF: In vitro fertilization; KEGG: Kyoto Encyclopedia of Genes and Genomes; LH: Luteinizing hormone; LIF: Leukemia inhibitory factor; LIFR: Leukemia inhibitory factor receptor; OMIM: Online Mendelian Inheritance in Man; PCOS: Polycystic ovary syndrome; PDN: Phenotypic Disease Network; POF: Premature ovarian failure; POI: Primary ovarian insufficiency; SHBG: Sex hormone-binding globulin; UMLS: Unified Medical Language System.

\section{Competing interests}

The authors declare that they have no competing interests.

\section{Authors' contributions}

JJT has been involved in conception and design, acquisition, analysis and interpretation of data, drafting the article and final approval of the version to be published. MAGP, TH and AC have been involved in analysis and interpretation of data, revising the article critically for important intellectual content and final approval of the version to be published.

\section{Author details}

${ }^{1}$ Department of Functional Biology and Physical Anthropology, Faculty of Biological Sciences, University of Valencia, Burjassot, Valencia 46100, Spain. ${ }^{2}$ Department of Genetics, Faculty of Biological Sciences, University of Valencia, Burjassot, Valencia 46100, Spain. ${ }^{3}$ Research Unit-INCLIVA, Hospital Clínico de Valencia, Valencia 46010, Spain. ${ }^{4}$ Department of Obstetrics and 
Gynecology, Keio University School of Medicine, Tokyo 160-8582, Japan. ${ }^{5}$ Department of Pediatrics, Obstetrics and Gynecology, Faculty of Medicine, University of Valencia, Valencia 46010, Spain. ${ }^{6}$ Service of Obstetrics and Gynecology, University Clinic Hospital, Valencia 46010, Spain.

\section{Received: 29 January 2015 Accepted: 9 April 2015 Published online: 15 April 2015}

\section{References}

1. Unified Medical Language System (UMLS) metathesaurus. Available at: https://uts.nlm.nih.gov/home.html. Accessed October 27, 2014.

2. Thoma ME, MCLain AC, Louis JF, King RB, Trumble AC, Sundaram R, et al. Prevalence of infertility in the United States as estimated by the current duration approach and a traditional constructed approach. Fertil Steril. 2013;99:1324-31.e1.

3. Tarín JJ, García-Pérez MA, Cano A. Assisted reproductive technology results: why are live-birth percentages so low? Mol Reprod Dev. 2014;81:568-83.

4. Liu CC, Tseng YT, Li W, Wu CY, Mayzus I, Rzhetsky A, et al. DiseaseConnect: a comprehensive web server for mechanism-based disease-disease connections. Nucleic Acids Res. 2014;42:W137-46.

5. DiseaseConnect web server. Available at: http://disease-connect.org. Accessed October 23, 2014

6. Oti M, Huynen MA, Brunner HG. Phenome connections. Trends Genet. 2008;24:103-6.

7. Tomao F, Lo Russo G, Spinelli GP, Stati V, Prete AA, Prinzi N, et al. Fertility drugs, reproductive strategies and ovarian cancer risk. J Ovarian Res. 2014;7:51.

8. Rizzuto I, Behrens RF, Smith LA. Risk of ovarian cancer in women treated with ovarian stimulating drugs for infertility. Cochrane Database Syst Rev. 2013:8, CD008215.

9. Schüler S, Ponnath M, Engel J, Ortmann O. Ovarian epithelial tumors and reproductive factors: a systematic review. Arch Gynecol Obstet. 2013;287:1187-204.

10. Zreik TG, Mazloom A, Chen Y, Vannucci M, Pinnix CC, Fulton S, et al. Fertility drugs and the risk of breast cancer: a meta-analysis and review. Breast Cancer Res Treat. 2010;124:13-26.

11. Siristatidis C, Sergentanis TN, Kanavidis P, Trivella M, Sotiraki M, Mavromatis I, et al. Controlled ovarian hyperstimulation for IVF: impact on ovarian, endometrial and cervical cancer-a systematic review and meta-analysis. Hum Reprod Update. 2013;19:105-23.

12. Sergentanis TN, Diamantaras AA, Perlepe C, Kanavidis P, Skalkidou A, Petridou ET. IVF and breast cancer: a systematic review and meta-analysis. Hum Reprod Update. 2014;2:106-23.

13. Centers for Disease Control and Prevention. Assisted Reproductive Technology, Fertility Clinic Success Rates Report; 2012. Available at: http://www.cdc.gov/art/reports/2012/fertility-clinic.html. Accessed October 1, 2014.

14. Kyoto Encyclopedia of Genes and Genomes (KEGG) pathway database. Available at: http://www.genome.jp/kegg/pathway.html. Accessed October 23, 2014

15. Hotaling JM, Walsh TJ. Male infertility: a risk factor for testicular cancer. Nat Rev Urol. 2009;6:550-6.

16. Peng $X$, Zeng $X$, Peng S, Deng D, Zhang J. The association risk of male subfertility and testicular cancer: a systematic review. PLoS One. 2009;4, e5591.

17. Walsh TJ. Male reproductive health and prostate cancer risk. Curr Opin Urol. 2011;21:506-13.

18. Eisenberg ML, Li S, Behr B, Cullen MR, Galusha D, Lamb DJ, et al. Semen quality, infertility and mortality in the USA. Hum Reprod. 2014:29:1567-74.

19. Belloc S, Cohen-Bacrie M, Amar E, Izard V, Benkhalifa M, Dalléac A, et al. High body mass index has a deleterious effect on semen parameters except morphology: results from a large cohort study. Fertil Steril. 2014;102:1268-73.

20. Omu AE. Sperm parameters: paradigmatic index of good health and longevity. Med Princ Pract. 2013;22 Suppl 1:30-42.

21. Sermondade N, Faure C, Fezeu L, Shayeb AG, Bonde JP, Jensen TK, et al. $\mathrm{BMI}$ in relation to sperm count: an updated systematic review and collaborative meta-analysis. Hum Reprod Update. 2013;19:221-31.

22. Eisenberg ML, Kim S, Chen Z, Sundaram R, Schisterman EF, Buck Louis GM. The relationship between male BMI and waist circumference on semen quality: data from the LIFE study. Hum Reprod. 2014;29:193-200.
23. Li Y, Lin H, Li Y, Cao J. Association between socio-psycho-behavioral factors and male semen quality: systematic review and meta-analyses. Fertil Steril. 2011;95:116-23.

24. Ely JJ. Inadequate levels of essential nutrients in developed nations as a risk factor for disease: a review. Rev Environ Health. 2003;18:111-29.

25. Leon DA, Shkolnikov VM, McKee M. Alcohol and Russian mortality: a continuing crisis. Addiction. 2009;104:1630-6.

26. Pilgrim $\mathrm{J}$, Woodford $\mathrm{N}$, Drummer $\mathrm{OH}$. Cocaine in sudden and unexpected death: a review of 49 post-mortem cases. Forensic Sci Int. 2013;227:52-9.

27. Rizzuto D, Fratiglioni L. Lifestyle factors related to mortality and survival: a mini-review. Gerontology. 2014;60:327-35.

28. Povey AC, Clyma JA, McNamee R, Moore HD, Baillie H, Pacey AA, et al. Modifiable and non-modifiable risk factors for poor semen quality: a case-referent study. Hum Reprod. 2012;27:2799-806.

29. Pacey AA, Povey AC, Clyma JA, McNamee R, Moore HD, Baillie H, et al. Modifiable and non-modifiable risk factors for poor sperm morphology. Hum Reprod. 2014;29:1629-36.

30. Permuth-Wey J, Besharat A, Sellers TA. Epidemiology of ovarian cancer: an update. In: Farghaly SA, editor. Advances in Diagnosis and Management of Ovarian Cancer. New York: Springer Science \& Business Media; 2014. p. 1-21.

31. Parikh $\mathrm{NI}$, Cnattingius S, Mittleman MA, Ludvigsson JF, Ingelsson E. Subfertility and risk of later life maternal cardiovascular disease. Hum Reprod. 2012;27:568-75.

32. Venn A, Hemminki E, Watson L, Bruinsma F, Healy D. Mortality in a cohort of IVF patients. Hum Reprod. 2001;16:2691-6.

33. Braat DD, Schutte JM, Bernardus RE, Mooij TM, van Leeuwen FE. Maternal death related to IVF in the Netherlands 1984-2008. Hum Reprod. 2010;25:1782-6.

34. Westerlund E, Brandt L, Hovatta O, Wallén H, Ekbom A, Henriksson P. Incidence of hypertension, stroke, coronary heart disease, and diabetes in women who have delivered after in vitro fertilization: a population-based cohort study from Sweden. Fertil Steril. 2014;102:1096-102.

35. Udell JA, Lu H, Redelmeier DA. Long-term cardiovascular risk in women prescribed fertility therapy. J Am Coll Cardiol. 2013;62:1704-12.

36. Yli-Kuha AN, Gissler M, Klemetti R, Luoto R, Hemminki E. Cancer morbidity in a cohort of 9175 Finnish women treated for infertility. Hum Reprod. 2012;27:1149-55

37. Calogero AE, Calabrò V, Catanuso M, Condorelli RA, La Vignera S. Understanding polycystic ovarian syndrome pathogenesis: an updated of its genetic aspects. J Endocrinol Invest. 2011;34:630-44.

38. Teede H, Deeks A, Moran L. Polycystic ovary syndrome: a complex condition with psychological, reproductive and metabolic manifestations that impacts on health across the lifespan. BMC Med. 2010:8:41.

39. Rotterdam ESHRE/ASRM-Sponsored PCOS consensus workshop group. Revised 2003 consensus on diagnostic criteria and long-term health risks related to polycystic ovary syndrome (PCOS). Hum Reprod. 2004;19:41-7.

40. CORUM - the Comprehensive Resource of Mammalian Protein Complexes. Available at: http://mips.helmholtz-muenchen.de/genre/proj/corum/index. html. Accessed October 28, 2014.

41. Kar S. Anthropometric, clinical, and metabolic comparisons of the four Rotterdam PCOS phenotypes: a prospective study of PCOS women. J Hum Reprod Sci. 2013;6:194-200.

42. Anderson SA, Barry JA, Hardiman PJ. Risk of coronary heart disease and risk of stroke in women with polycystic ovary syndrome: A systematic review and meta-analysis. Int J Cardiol. 2014;176:486-7.

43. Vink JM, Sadrzadeh S, Lambalk CB, Boomsma DI. Heritability of polycystic ovary syndrome in a Dutch twin-family study. J Clin Endocrinol Metab. 2006;91:2100-4.

44. Hunter A, Vimplis S, Sharma A, Eid N, Atiomo W. To determine whether first-degree male relatives of women with polycystic ovary syndrome are at higher risk of developing cardiovascular disease and type II diabetes mellitus. J Obstet Gynaecol. 2007;27:591-6.

45. Davies MJ, Marino JL, Willson KJ, March WA, Moore VM. Intergenerational associations of chronic disease and polycystic ovary syndrome. PLoS One. 2011;6, e25947.

46. Louwers YV, Roest-Schalken ME, Kleefstra N, Roeters van Lennep J, van den Berg M, Fauser BC, et al. Excess mortality in mothers of patients with polycystic ovary syndrome. Hum Reprod. 2014;29:1780-6.

47. Savaris RF, Groll JM, Young SL, DeMayo FJ, Jeong JW, Hamilton AE, et al. Progesterone resistance in PCOS endometrium: a microarray analysis in clomiphene citrate-treated and artificial menstrual cycles. J Clin Endocrinol Metab. 2011;96:1737-46. 
48. Ali AT. Risk factors for endometrial cancer. Ceska Gynekol. 2013;78:448-59.

49. Chittenden BG, Fullerton G, Maheshwari A, Bhattacharya S. Polycystic ovary syndrome and the risk of gynaecological cancer: a systematic review. Reprod Biomed Online. 2009;19:398-405.

50. Barry JA, Azizia MM, Hardiman PJ. Risk of endometrial, ovarian and breast cancer in women with polycystic ovary syndrome: a systematic review and meta-analysis. Hum Reprod Update. 2014;20:748-58.

51. Ali AT. Reproductive factors and the risk of endometrial cancer. Int J Gynecol Cancer. 2014;24:384-93.

52. Housman E, Reynolds RV. Polycystic ovary syndrome: a review for dermatologists: Part I. Diagnosis and manifestations. J Am Acad Dermatol. 2014;71:847.e1-e10.

53. Daan NM, Louwers YV, Koster MP, Eijkemans MJ, de Rijke YB, Lentjes EW, et al. Cardiovascular and metabolic profiles amongst different polycystic ovary syndrome phenotypes: who is really at risk? Fertil Steril. 2014;102:1444-51.e3.

54. Lambrinoudaki I. Cardiovascular risk in postmenopausal women with the polycystic ovary syndrome. Maturitas. 2011;68:13-6.

55. Silva CA, Yamakami LY, Aikawa NE, Araujo DB, Carvalho JF, Bonfá E. Autoimmune primary ovarian insufficiency. Autoimmun Rev. 2014;13:427-30.

56. Nelson LM. Clinical practice. Primary ovarian insufficiency. N Engl J Med. 2009:360:606-14

57. Maclaran K, Horner E, Panay N. Premature ovarian failure: long-term sequelae. Menopause Int. 2010;16:38-41.

58. Cox L, Liu JH. Primary ovarian insufficiency: an update. Int J Womens Health. 2014;6:235-43.

59. Shuster LT, Rhodes DJ, Gostout BS, Grossardt BR, Rocca WA. Premature menopause or early menopause: long-term health consequences. Maturitas. 2010;65:161-6

60. Li S, Rosenberg L, Wise LA, Boggs DA, LaValley M, Palmer JR. Age at natural menopause in relation to all-cause and cause-specific mortality in a follow-up study of US black women. Maturitas. 2013;75:246-52.

61. Shah D, Nagarajan N. Premature menopause - Meeting the needs. Post Reprod Health. 2014;20:62-8.

62. Mahendru AA, Morris E. Cardiovascular disease in menopause: does the obstetric history have any bearing? Menopause Int. 2013;19:115-20.

63. Scott EL, Zhang QG, Vadlamudi RK, Brann DW. Premature menopause and risk of neurological disease: basic mechanisms and clinical implications. Mol Cell Endocrinol. 2014;389:2-6.

64. Atwood CS, Bowen RL. The reproductive-cell cycle theory of aging: an update. Exp Gerontol. 2011:46:100-7.

65. Greene AD, Patounakis G, Segars JH. Genetic associations with diminished ovarian reserve: a systematic review of the literature. J Assist Reprod Genet. 2014;31:935-46.

66. Society for Assisted Reproductive Technology (SART). 2012 National Clinic Summary Report. Available at: http://sart.org/find_frm.html. Accessed October 3, 2014

67. Richardson MC, Guo M, Fauser BC, Macklon NS. Environmental and developmental origins of ovarian reserve. Hum Reprod Update. 2014;20:353-69.

68. Gleicher N, Kim A, Weghofer A, Kushnir VA, Shohat-Tal A, Lazzaroni E, et al. Hypoandrogenism in association with diminished functional ovarian reserve. Hum Reprod. 2013;28:1084-91.

69. Verit FF, Keskin S, Omer B, Yalcinkaya S, Sakar N. Is there any relationship between cardiovascular risk markers and young women with diminished ovarian reserve? Gynecol Endocrinol. 2014;30:697-700.

70. Titus S, Li F, Stobezki R, Akula K, Unsal E, Jeong K, et al. Impairment of BRCA 1-related DNA double-strand break repair leads to ovarian aging in mice and humans. Sci Transl Med. 2013;5:172ra21.

71. Wang ET, Pisarska MD, Bresee C, Ida Chen YD, Lester J, Afshar Y, et al. BRCA1 germline mutations may be associated with reduced ovarian reserve. Fertil Steril. 2014;102:1723-8.

72. Brohet RM, Velthuizen ME, Hogervorst FB, Meijers-Heijboer HE, Seynaeve C, Collée MJ, et al. HEBON Resource. Breast and ovarian cancer risks in a large series of clinically ascertained families with a high proportion of BRCA1 and BRCA2 Dutch founder mutations. J Med Genet. 2014;51:98-107.

73. Mersch J, Jackson MA, Park M, Nebgen D, Peterson SK, Singletary C, et al. Cancers associated with BRCA1 and BRCA2 mutations other than breast and ovarian. Cancer. 2015;121:269-75.

74. Oktay K, Kim JY, Barad D, Babayev SN. Association of BRCA1 mutations with occult primary ovarian insufficiency: a possible explanation for the link between infertility and breast/ovarian cancer risks. J Clin Oncol. 2010;28:240-4
75. Rzepka-Górska I, Tarnowski B, Chudecka-Głaz A, Górski B, Zielińska D, Tołoczko-Grabarek A. Premature menopause in patients with BRCA1 gene mutation. Breast Cancer Res Treat. 2006;100:59-63.

76. Finch A, Valentini A, Greenblatt E, Lynch HT, Ghadirian P, Armel S, et al. Frequency of premature menopause in women who carry a BRCA1 or BRCA2 mutation. Fertil Steril. 2013:99:1724-8.

77. Lin WT, Beattie M, Chen LM, Oktay K, Crawford SL, Gold EB, et al. Comparison of age at natural menopause in BRCA1/2 mutation carriers with a non-clinicbased sample of women in northern California. Cancer. 2013;119:1652-9.

78. Collins IM, Milne RL, McLachlan SA, Friedlander M, Hickey M, Weideman PC, et al. Do BRCA1 and BRCA2 mutation carriers have earlier natural menopause than their noncarrier relatives? Results from the Kathleen Cuningham Foundation Consortium for Research into Familial Breast Cancer. J Clin Oncol. 2013;31:3920-5.

79. Oktay K, Moy F, Titus S, Stobezki R, Turan V, Dickler M, et al. Age-related decline in DNA repair function explains diminished ovarian reserve, earlier menopause, and possible oocyte vulnerability to chemotherapy in women with BRCA mutations. J Clin Oncol. 2014;32:1093-4.

80. Batt RE, Martin DC, Odunsi K. Endometriosis of the retrocervical septum is proposed to replace the anatomically incorrect term endometriosis of the rectovaginal septum. Hum Reprod. 2014;29:2603-5.

81. Munksgaard PS, Blaakaer J. The association between endometriosis and gynecological cancers and breast cancer: a review of epidemiological data. Gynecol Oncol. 2011;123:157-63.

82. Pavlidou A, Vlahos NF. Endometriosis and ovarian cancer: clinical and molecular aspects. Minerva Endocrinol. 2014;39:155-65.

83. Nezhat FR, Pejovic T, Reis FM, Guo SW. The link between endometriosis and ovarian cancer: clinical implications. Int J Gynecol Cancer. 2014;24:623-8.

84. Vlahos NF, Economopoulos KP, Fotiou S. Endometriosis, in vitro fertilization and the risk of gynaecological malignancies, including ovarian and breast cancer. Best Pract Res Clin Obstet Gynaecol. 2010;24:39-50.

85. Kim HS, Kim TH, Chung HH, Song YS. Risk and prognosis of ovarian cancer in women with endometriosis: a meta-analysis. Br J Cancer. 2014;110:1878-90.

86. Hu X, Zhou Y, Feng Q, Wang R, Su L, Long J, et al. Association of endometriosis risk and genetic polymorphisms involving biosynthesis of sex steroids and their receptors: an updating meta-analysis. Eur J Obstet Gynecol Reprod Biol. 2012;164:1-9.

87. Forte A, Cipollaro M, Galderisi U. Genetic, epigenetic and stem cell alterations in endometriosis: new insights and potential therapeutic perspectives. Clin Sci (Lond). 2014;126:123-38.

88. Rahmioglu N, Nyholt DR, Morris AP, Missmer SA, Montgomery GW, Zondervan KT. Genetic variants underlying risk of endometriosis: insights from meta-analysis of eight genome-wide association and replication datasets. Hum Reprod Update. 2014;20:702-16.

89. Worley MJ, Welch WR, Berkowitz RS, Ng SW. Endometriosis-associated ovarian cancer: a review of pathogenesis. Int J Mol Sci. 2013;14:5367-79.

90. Siufi Neto J, Kho RM, Siufi DF, Baracat EC, Anderson KS, Abrão MS. Cellular, histologic, and molecular changes associated with endometriosis and ovarian cancer. J Minim Invasive Gynecol. 2014;21:55-63.

91. Cetin I, Cozzi V, Antonazzo P. Infertility as a cancer risk factor - a review. Placenta. 2008:29 Suppl B:169-77.

92. Pearce CL, Templeman C, Rossing MA, Lee A, Near AM, Webb PM, et al. Association between endometriosis and risk of histological subtypes of ovarian cancer: a pooled analysis of case-control studies. Lancet Oncol. 2012;13:385-94.

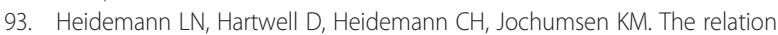
between endometriosis and ovarian cancer - a review. Acta Obstet Gynecol Scand. 2014;93:20-31.

94. Venn A, Watson L, Bruinsma F, Giles G, Healy D. Risk of cancer after use of fertility drugs with in-vitro fertilisation. Lancet. 1999;354:1586-90.

95. Ness RB, Cramer DW, Goodman MT, Kjaer SK, Mallin K, Mosgaard BJ, et al. Infertility, fertility drugs, and ovarian cancer: a pooled analysis of case-control studies. Am J Epidemiol. 2002;155:217-24.

96. Venn A, Healy D, McLachlan R. Cancer risks associated with the diagnosis of infertility. Best Pract Res Clin Obstet Gynaecol. 2003;17:343-67.

97. G S, A B, Kamath A, Shivaprakash P, Adhikari P, Up R, et al. Acanthosis nigricans in PCOS patients and its relation with type 2 diabetes mellitus and body mass at a tertiary care hospital in Southern India. J Clin Diagn Res. 2013;7:317-9.

98. Xie J, Kvaskoff M, Li Y, Zhang M, Qureshi AA, Missmer SA, et al. Severe teenage acne and risk of endometriosis. Hum Reprod. 2014;29:2592-9. 
99. Shah DK, Correia KF, Vitonis AF, Missmer SA. Body size and endometriosis: results from 20 years of follow-up within the Nurses' Health Study II prospective cohort. Hum Reprod. 2013;28:1783-92.

100. Vercellini P, Buggio L, Somigliana E, Barbara G, Viganò P, Fedele L. Attractiveness of women with rectovaginal endometriosis: a case-control study. Fertil Steril. 2013;99:212-8.

101. Vercellini P, Buggio L, Somigliana E, Dridi D, Marchese MA, Viganò P. 'Behind blue eyes': the association between eye colour and deep infiltrating endometriosis. Hum Reprod. 2014;29:2171-5.

102. Buggio L, Vercellini P, Somigliana E, Viganò P, Frattaruolo MP, Fedele L. "You are so beautiful": behind women's attractiveness towards the biology of reproduction: a narrative review. Gynecol Endocrinol. 2012;28:753-7.

103. Atkinson J, Pipitone RN, Sorokowska A, Sorokowski P, Mberira M, Bartels A, et al. Voice and handgrip strength predict reproductive success in a group of indigenous African females. PLoS One. 2012;7, e41811.

104. Arden R, Gottfredson LS, Miller G, Pierce A. Intelligence and semen quality are positively correlated. Intelligence. 2009;37:277-82

105. Soler C, Kekäläinen J, Núñez M, Sancho M, Álvarez JG, Núñez J, et al. Male facial attractiveness and masculinity may provide sex- and culture-independent cues to semen quality. J Evol Biol. 2014;27:1930-8.

106. Peters M, Rhodes G, Simmons LW. Does attractiveness in men provide clues to semen quality? J Evol Biol. 2008;21:572-9.

\section{Submit your next manuscript to BioMed Central and take full advantage of:}

- Convenient online submission

- Thorough peer review

- No space constraints or color figure charges

- Immediate publication on acceptance

- Inclusion in PubMed, CAS, Scopus and Google Scholar

- Research which is freely available for redistribution 